\title{
Microstructural effects of banana leaf on adhesion and self cleaning behaviour
}

\author{
Hasrawati Abu Hassan ${ }^{1}$, Mariyam Jameelah Ghazali ${ }^{1, a}$, Che Husna Azhari ${ }^{1}$ and Cevdet Meric ${ }^{2}$ \\ ${ }^{1}$ Department of Mechanical and Materials Engineering, Faculty of Engineering and Built Environment, Universiti Kebangsaan Malaysia, \\ 43600 UKM Bangi, Selangor, Malaysia \\ ${ }^{2}$ Faculty of Engineering, Fatih University, 34500 Buyukcekmece/lstanbul/Turkey
}

\begin{abstract}
A recent discovery on banana leaf that has high potential as the lotus effect has intrigued further investigation particularly on the mechanism of its self-cleaning behaviour. In this paper, the adhesive and the contact angle effects of the banana leaf, named Musa Parasidiaca L. (berangan) surface in determining the self-cleaning effect is discussed especially at high temperatures. Samples were slid against a heated metal plate at two different temperatures; $100^{\circ} \mathrm{C}$ and $200^{\circ} \mathrm{C}$, under different loads; $0.5 \mathrm{~kg}$ and $1 \mathrm{~kg}$. Correlation between the leaf morphologies, adhesion force and contact angles were discussed. Measurements were carefully carried out by using the various pressure scanning electron microscopy (VP-SEM), an atomic force microscope (AFM) and a drop shape analysis (DSA). The adhesion force and contact angle were decreased at the highest temperature and load. The adhesion force was found to be reduced from $5.6 \mathrm{nN}$ to $1.9 \mathrm{nN}$ and the value of contact angles was decreased from $108.9^{\circ}$ to $99.4^{\circ}$. In short, it is hope that the new discovery of this study can shed light on its importance to mankind particularly in surface modifications for applications at higher temperature.
\end{abstract}

\section{Introduction}

Over recent years, there are several interests in nature that include many plants and animals exhibit superhydrophobic property with water contact angle (WCA) more than $150^{\circ}$ and sliding angle (SA) lest than $10^{\circ}$, such as lotus leaves, rice leaves, red rose petals, gecko feet and desert beetle [1]. Lotus effect occurs when falling water drops onto the leaves can bead up and roll off, washing dirt from the lotus leaves so that they are self-cleaning [2].

Lotus leaf is a typical natural super-hydrophobic surface with WCA and SA on the surface of lotus leaf is about $161.0 \pm 2.7^{\circ}$ and $2^{\circ}$, respectively because of the hierarchy surface structures [3]. Figure 1 shows a scanning electron micrograph (SEM) image of the lotus leaf surface where papillas with branch-like nanostructures were found on this surface in a random distribution efficiently increases the roughness.

Hydrophobicity of leaf is due to the surface that contained a layer of hydrophobic wax and also surface topography [4]. Wax is one of the materials that can construct hydrophobic (water-repellent) surfaces [5]. The existence of wax will increase the surface roughness and contact angle.

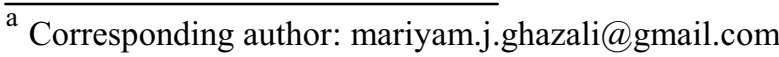
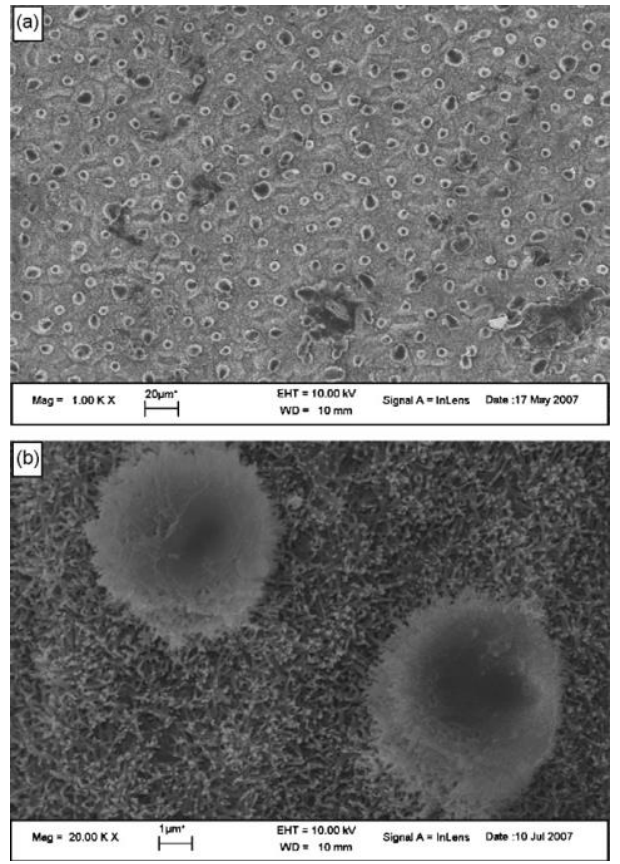

Figure 1. (a) Large-area SEM image of the surface of lotus leaf. (b) Enlarged view of lotus leaf from (a)[3].

The wettability of plant surfaces may change with age and maturity with a little variation in contact angle [6]. In this study, the leaf is taken from the plants age between 7 to 9 months and the layer number 3 down the profile [7]. 
Meaning that the leaf is selected from the third layer of the banana leaves counted from upper part of the plant. The size and distribution of wax differed among the types of banana. The irregular shape of wax deposits form walls that may create the air pocket that contribute to the surface roughness and wettability.

Even though lotus and rose petal have the superhydrophobic behaviour, but both show different adhesive force [8]. The reason why they have different adhesive forces is that the pitch values of microstructures and the density of nanostructures of their surfaces are varies, thus giving rise to different states of water droplet on the two surfaces.

Recently, the effect of high temperature and loading condition on the plant surface is not yet discover. This paper is comparing the results of two different temperatures $\left(100^{\circ} \mathrm{C}\right.$ and $\left.200^{\circ} \mathrm{C}\right)$ and two different applied loads $(0.5 \mathrm{~kg}$ and $1.0 \mathrm{~kg})$. The purpose of this paper is to stimulate the further investigation on the banana leaf as similar to lotus and other plants surface that have been established.

\section{Methology}

\subsection{Starting Materials}

A dessert type of banana, Musa Parasidiaca L. (Berangan) was collected from Taman Pertanian Universiti (TPU), Universiti Pertanian Malaysia to obtain the standard age and leave taken form the banana cultivar. Leaves were sectioned to $20 \mathrm{~cm} \times 20 \mathrm{~cm}$ and ready for the next steps.

\subsection{Sliding Test}

Metal plates with two different loads $(0.5 \mathrm{~kg}$ and $1.0 \mathrm{~kg})$ were heated in a furnace at two different temperatures $\left(100^{\circ} \mathrm{C}\right.$ and $\left.200^{\circ} \mathrm{C}\right)$ as a medium that acted similar as the typical home iron. The parameters were selected as a continuity from the study that have been done using typical home iron [9]. Sliding were conducted constantly for 20 s on the adaxial (top) of the banana leave. Adaxial side is selected because it is exposed to the environment and during the ironing work; the adaxial side is attached to the iron.

\subsection{Microstructure and Surface Examination}

Banana leaf samples were prepared for detailed microstructural observations under a scanning electron microscope (LEO 1450VP). Prior to observations, samples were rinsed in $0.1 \mathrm{M}$ sodium cacodylate buffer, dehydrated in a graded series of ethanol, and then dried in hexametyldisilazano. The dried samples were mounted on stubs and stored in dry cabinet before being coated with gold and examined.

\subsection{Adhesion Force and Surface Roughness}

A commercial Atomic Force Microscope (Park System) with contact cantilever (PPP-CONTSCR 10M) is used to obtain the surface roughness and adhesion force of the treated banana leave. The surface roughness is obtained from the amount of bumps and the adhesion force was determined from the pull-off force of the force distance curve measured with the AFM [10].

\subsection{Contact Angle Measurement}

Samples are placed horizontally on glass slides. Water contact angle is determined as per standard ASTM D7334 using Drop Shape Analysis.

\section{Result and Discussion}

\subsection{Microstructure and surface examination}

Figure 2 shows the SEM results of the banana leaf surface.

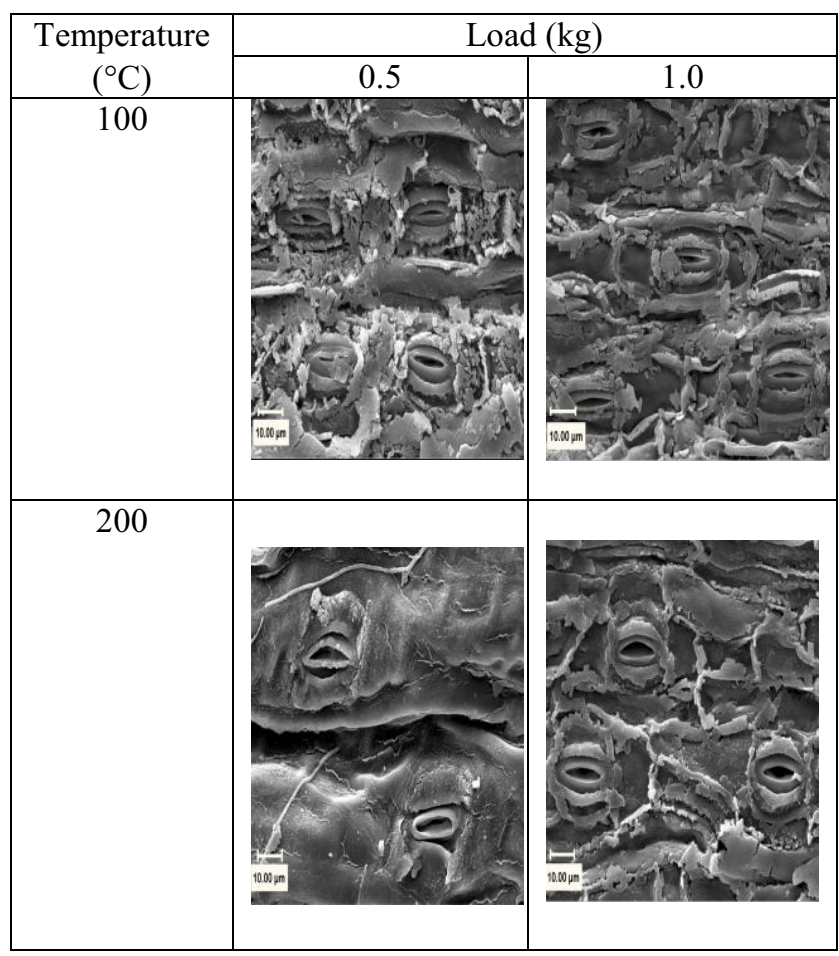

Figure 2. SEM images of the banana leaf surface (Berangan) at different load and temperature.

The melting point of wax that is below $100^{\circ} \mathrm{C}$ did show in the micrograph in Figure 2. Even the wax is melted, the form of air pocket from the wax wall still maintain till $200^{\circ} \mathrm{C}$. The formation of air pocket contributes to the hydrophobic and self-cleaning behavior of banana leaf.

\subsection{Adhesion Force and Surface Roughness}

Figure 3 shows that the adhesion force is decrease with the increasing of temperature, same as the load applied. The applied loads have compressed the wall of the air pocket and lower the adhesion force of the surface. The higher temperature also lowers the force as well. Melted 
wax caused the adhesion to decrease. Hydrophobicity surface includes low adhesion, high contact angle and high surface roughness [5].

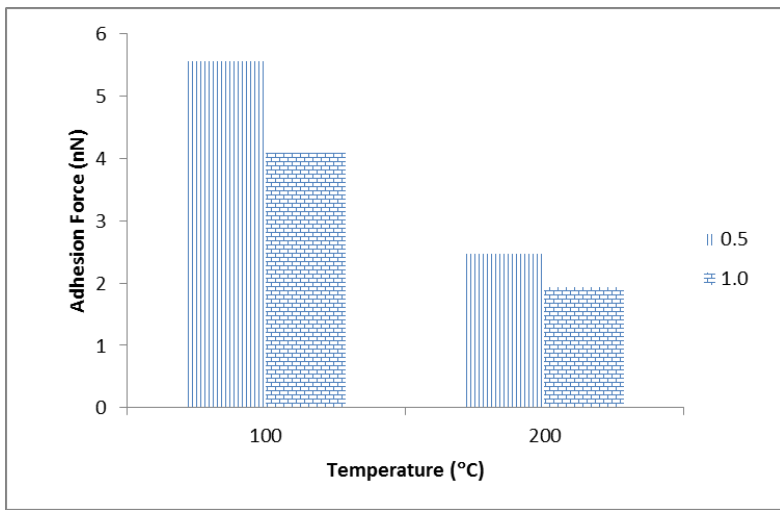

Figure 3 Graph shows adhesion force for Musa Parasidiaca L. (Berangan) at different load and temperature.

Surface roughness is shown in Figure 4. Melting of wax caused the roughness of the surface to increase for the lower load and decrease in the higher one.

In order to determine the adhesion force, contact tip is use. As the principle to get adhesion force is from the pull-off force of the curve measured, at higher temperature and load, the wax is melted and compressed. When the tip comes into contact with the leaf area, the sample is not easily deformed as the moisture is gone. This will cause the leaf to dry and give a rougher surface when the temperature is increase. For the higher load, the decrease in the surface roughness may cause by higher load that smoothing the surface.

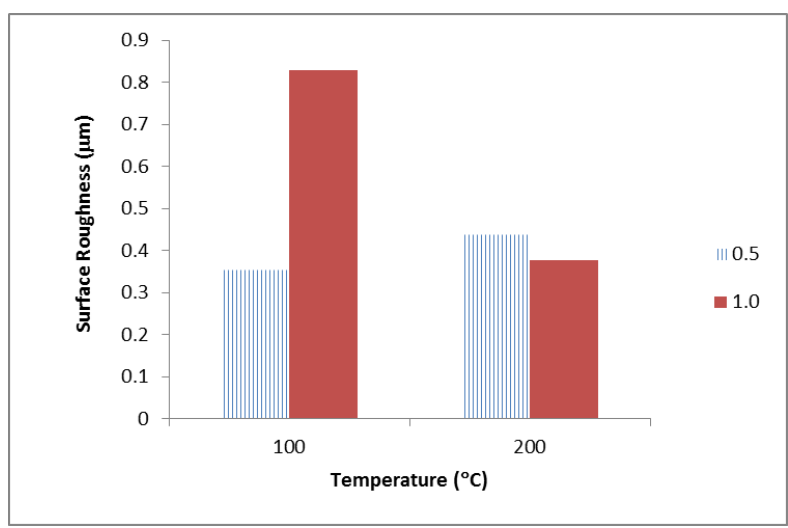

Figure 4 Graph shows surface roughness for Musa Parasidiaca L. (Berangan) at different load and temperature

\subsection{Contact Angle}

The continuity from the results of the micrograph and adhesion force showed that temperature and load also affects the wettability of the surface. Value of contact angle also exhibit reduction as the increment of temperature and load. Decrease in contact angle due to the melting of wax and minimized the rough surface that contribute to the wetting behaviour. The value of contact angle is shown in Figure 5.

\begin{tabular}{|c|c|c|}
\hline \multirow{2}{*}{$\begin{array}{c}\text { Temperature } \\
\left({ }^{\circ} \mathrm{C}\right)\end{array}$} & \multicolumn{2}{|c|}{ Load (kg) } \\
\hline & 0.5 & 1.0 \\
\hline \multicolumn{3}{|l|}{100} \\
\hline & $108.9 \pm 0.31$ & $107.1 \pm 0.29$ \\
\hline \multicolumn{3}{|l|}{200} \\
\hline & $101.6 \pm 0.17$ & $99.4 \pm 0.95$ \\
\hline
\end{tabular}

Figure 5. Images shows contact angle for Musa Parasidiaca L. (Berangan) at different load and temperature.

\section{Conclusions}

Considering all of this evidence, it seems that at $100^{\circ} \mathrm{C}$ and $200^{\circ} \mathrm{C}$ with applied loads $0.5 \mathrm{~kg}$ and $1.0 \mathrm{~kg}$ did not affect much in micrograph and contact angle as the form of stomata is still obvious in the macrograph. The adhesion force and surface roughness is donated by the deformation of the wax after applying certain load and temperature. Even though the melting point is lower than $100^{\circ} \mathrm{C}$, the waxes are still there giving the hydrophobic behavior as the contact angle still give the reading more than $90^{\circ}$. Further research should focus on another applied temperatures and loads to determine the feasibility of banana leaves as a bio-adhesion lubricant at high temperature.

\section{Acknowledgement}

This work was financially supported by FRGS/2/2013/TK04/UKM/02/2 and the authors are thankful to Prof Dr Yakub Bakis, Coordinator of BioNano Technology Centre and Dr. Isa Seker from Fatih University, Istanbul for all their full supports.

\section{References}

1. J. Liu, X. Xiao, Y. Shi, C. Wan. Liu, J., Xiao X., Shi, Y., C. Wan, Appl. Surf. Sc., 297 (2014)

2. M. Zhang, S. Feng, L. Wang and Y. Zheng, Biotribology, 5 (2016)

3. W. Xi, Z. Qiao, C. Zhu, A. Jia, A and M. Li, Applied Surface Science, 255 (2009)

4. J. Wang, H. Chen, T. Sui, A. Li and D. Chen, Plant Science, 176 (2009)

5. Z. Burton, and B. Bhushan, Ultramicroscopy, 106 (2006)

6. R.E. Gaskin, K.D. Steele and W.A. Forster,(2005). New Zealand Plant Protection 58 (2005) 
7. W. Zakaria and A.R. Razak, J. Trop. Agric. and Fd. Sc. 27, 2 (1999)

8. G. Wang, Z. Guo and W. Liu, Journal of Bionic Engineering, 11 (2014)

9. F. Azura, Final Year Theses (Universiti Kebangsaan Malaysia, 2014)

10. K. Meine, K. Kloß, T. Schneider, T. and D. Spaltmann, D. (2004) Surf. Interface Anal. 36 (2004) 